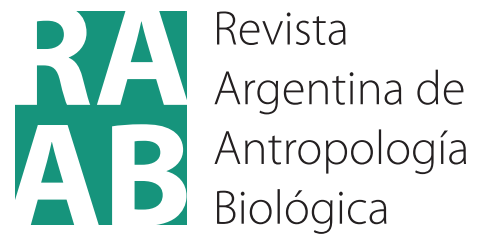

TRABAJO ORIGINAL

\section{Agrupamientos poblacionales en Uruguay inferidos en base a la distribución de apellidos}

\section{Population groups in Uruguay based on the distribution of surnames}

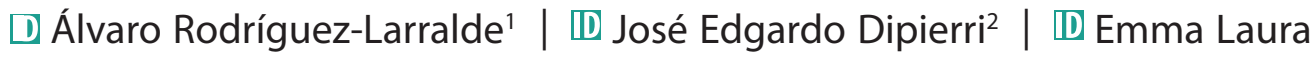 \\ Alfaro $^{2,3}$ | ID Mónica Sans ${ }^{4 *}$
}

1) Centro de Medicina Experimental. Laboratorio de Genética Humana. IVIC. Caracas. Venezuela. 2) Departamento de Genética y Bioantropología. Instituto de Biología de la Altura. Universidad Nacional de Jujuy. San Salvador de Jujuy. Argentina. 3) Instituto de Ecorregiones Andinas (INECOA). Consejo Nacional de Investigaciones Científicas y Técnicas - Universidad Nacional de Jujuy. San Salvador de Jujuy. Argentina. 4) Departmento de Antropología Biológica. Facultad de Humanidades y Ciencias de la Educación. Universidad de la República. Montevideo. Uruguay.

\section{REVISTA ARGENTINA DE \\ ANTROPOLOGÍA BIOLÓGICA \\ Volumen 23, Número 1}

Enero-Junio 2021

\section{Financiamiento:}

Agencia Nacional de Promoción Científica y Tecnológica (ANPCyT PICT 2015-2797) y Secretaría de Ciencia, Técnica y Estudios Regionales, Universidad Nacional de Jujuy (SECTER - UNJu 08/F032) otorgados a Emma L. Alfaro.

*Correspondencia a:

Mónica Sans. Departamento de Antropología Biológica, Facultad de Humanidades y Ciencias de la Educación. Universidad de la República. Magallanes 1577. 11200 Montevideo. Uruguay.E-mail:mbsans@gmail.com

RECIBIDO: 16 Diciembre 2019

ACEPTADO: 11 Mayo 2020

DOI: $10.24215 / 18536387 e 025$

\section{e-ISSN 1853-6387}

https://revistas.unlp.edu.ar/raab

\section{Resumen}

Recientemente publicamos el análisis de los apellidos del Padrón Electoral de 2010 de la República Oriental del Uruguay a través de distancias isonímicas, basado en sus 19 departamentos y las 171 comparaciones posibles entre estos, lo que mostró la formación de dos grandes agrupamientos en el sur, y otros pocos vinculados en el norte y este. El objetivo del presente trabajo es detallar el análisis de las distancias isonímicas utilizando la distribución de apellidos, no a nivel departamental, sino de los 221 municipios en los cuales se divide el país. Se estimaron las distancias isonímicas entre ellos, tomados dos a dos (24.310 comparaciones), se construyeron las matrices de distancia correspondientes y se correlacionaron con las de distancias geográficas. La correlación fue mayor para la distancia euclídea $(r=0,253 \pm 0,033)$, intermedia para la de Lasker $(r=0,18 \pm 0,029)$ y menor para la de Nei $(r=0,17 \pm 0,03)$, todas estadísticamente significativas $(p<0,001)$. Se construyeron nuevos dendrogramas determinándose agrupamientos no identificados previamente. Se destaca cierta homogeneidad en el sur con las menores distancias, con 7 agrupamientos cercanos a Montevideo. Al norte, con mayores distancias que denotan mayor heterogeneidad de apellidos, se identifican 8 agrupamientos. Se corrobora que el río Negro actuó como barrera geográfica, pero también se observan límites marcados por otros accidentes orográficos, hidrográficos, históricos y factores sociodemográficos no identificados en el análisis anterior. Por último, se resalta la utilidad del uso de apellidos para analizar la estructura poblacional, permitiendo el análisis global de la población de un país, en este caso, Uruguay. Rev Arg Antrop Biol 23(1), 2021. doi:10.24215/18536387e025

Palabras Clave: isonimia; distancia genética; agrupamientos regionales 


\begin{abstract}
Recently we published the analysis of surnames of the 2010 Electoral Register of República Oriental del Uruguay through calculation of isonymic distances, based on the 19 departments of the country, and the 171 possible comparisons, showing two large clusters in the south, and a few others with smaller relationships in the north and east. The purpose of the present work is to deepen the analysis of isonymic distances using the distribution of surnames based on the 221 municipalities, instead of departments, that make up the country. Isonymic distances were estimated taking two at a time, for 24,310 comparisons. Matrices of isonymic distances were tested for correlation with geographic distance. The correlations were higher $(r=0.253 \pm 0.033)$ for euclidean, intermediate for Lasker $(r=0.18 \pm 0.029)$, and lower for Nei's distance $(r=0.17 \pm 0.03)$, all statistically significant $(p<0.001)$. New dendrograms were constructed with clusters not previously determined. The relative surname homogeneity in the south is noteworthy, with 7 clusters close to Montevideo. In the north, with 8 clusters identified, distances are greater, revealing more surname heterogeneity. It was confirmed that the Río Negro acted as a geographical barrier, but orographic, hydrographic, historic, and sociodemographic factors, which were not detected in the previous analysis, were also involved. Finally, the usefulness of surnames in the analysis of the population structure of a whole country, in this case Uruguay, was highlighted. Rev Arg Antrop Biol 23(1), 2021. doi:10.24215/18536387e025
\end{abstract}

Keywords: isonymy; genetic distance; regional clusters

El estudio de las poblaciones humanas considerando aspectos biológicos o bioculturales ha sido abordado desde muy distintos puntos de vista y metodologías. En la actualidad, gran número de estudios se basan en análisis que incluyen marcadores moleculares. Sin embargo, en términos generales, estos estudios se ven generalmente limitados al análisis de pocas poblaciones o individuos, dados los costos y la dificultad de obtener muestras representativas de países o grandes regiones. Los estudios basados en apellidos, entre otros, permiten abarcar un gran número de individuos y analizar las relaciones entre ellos pero también entre las poblaciones que integran.

Los métodos y técnicas de análisis de apellidos se han ido perfeccionando. En 1965 Crow y Mange publican un trabajo (Crow y Mange, 1965) donde demuestran que en una población, bajo ciertas suposiciones, un cuarto de la frecuencia de matrimonios entre personas con el mismo apellido (matrimonios isonímicos) equivale al coeficiente de consanguinidad promedio; matemáticamente $\mathrm{F}=\mathrm{l} / 4$ donde $\mathrm{F}$ es el coeficiente de consanguinidad promedio de la población estudiada e I la frecuencia de uniones isonímicas observadas en ésta. Lasker (1977) propone un método para medir la relación biológica entre poblaciones a través de apellidos, llamando al parámetro correspondiente "coeficiente de parentesco". Valores altos de este coeficiente indican que las poblaciones son parecidas en cuanto a la composición de sus apellidos. Más recientemente, Rodríguez-Larralde y colaboradores proponen una variación del coeficiente de parentesco de Lasker para convertirlo en distancia (RodríguezLarralde et al., 1998). Otras medidas de distancias genéticas para inferir la relación entre poblaciones utilizando marcadores genéticos, como la euclídea (Cavalli-Sforza y Edwards, 1967) y la de Nei (Nei, 1973), también se han utilizado con frecuencias de apellidos.

Con el avance de la informática y el almacenamiento de enorme cantidad de información en forma digitalizada, se hacen disponibles bases de datos de los apellidos existentes 
en países o en amplias divisiones administrativas dentro de un país, como son los registros civiles, telefónicos y electorales. Estas comienzan a ser utilizadas para estimar la estructura genética de amplias regiones geográficas y la relación entre poblaciones a través de apellidos (Barrai, Scapoli, Mamolini, y Rodríguez-Larralde, 1999; Barrai, Rodríguez-Larralde, Mamolini, Manni y Scapoli, 2001; Dipierri et al., 2005; Rodríguez-Larralde et al., 1998; Rodríguez-Larralde y Castro de Guerra, 2012; Rodríguez-Larralde, Morales y Barrai, 2000; Rodríguez-Larralde, Scapoli, Mamolini y Barrai, 2007; Scapoli, Mamolini, Carrieri, RodríguezLarralde y Barrai, 2007; Tarskaia, Scapoli, Mamolini, Rodríguez-Larralde y Barrai, 2009; Zei, Matessi, Siri, Moroni y Cavalli-Sforza, 1983; entre muchos otros). Una revisión de los avances del uso de apellidos durante los últimos años del siglo pasado fue hecha por Colantonio y colegas (Colantonio, Lasker, Kaplan y Fuster, 2003).

En el año 2012 JED consiguió, a través de la Corte Electoral de la República Oriental del Uruguay, una copia de la lista electoral de 2010, para su uso en investigación exclusivamente. Recientemente, con información del mismo Padrón Electoral y utilizando distancias entre poblaciones basadas en apellidos (distancias isonímicas), presentamos resultados sobre la relación entre los 19 departamentos del Uruguay, con la finalidad de inferir la estructura resultante de movimientos poblacionales y deriva génica en el país (Carrieri et al., 2020). El objetivo del presente trabajo es profundizar en el análisis de las distancias isonímicas entre las poblaciones uruguayas, ya no a nivel de los 19 departamentos sino de los 221 municipios que lo integran, y por consecuencia, aumentando la cantidad de comparaciones de 171 a 24.310. A partir de este análisis, se espera inferir barreras geográficas y culturales que reflejen en detalle el comportamiento de las poblaciones.

\section{MATERIAL Y MÉTODOS}

A partir de la información del Padrón Electoral de 2010 se elaboraron bases de datos con la distribución de los apellidos de los 221 municipios que integran los 19 departamentos de la República Oriental del Uruguay, para un total de 2.501.776 individuos. Esa cifra corresponde al número de uruguayos con 18 o más años, que son el 76\% de la población de acuerdo a datos del Censo Nacional de 2011, el más próximo en el tiempo a los datos analizados (INE, 2011). El nombre de cada municipio, su departamento, así como el código utilizado para la identificación de los mismos en las figuras que se presentan, aparecen en la Tabla 1. Se incluye también el grupo al que se asignó cada municipio según el análisis que se describe en este trabajo. Los códigos de identificación consisten en 4 cifras, las 2 primeras (del 01 al 19) que corresponden al departamento según su orden alfabético, seguido de otros dos dígitos asignados secuencialmente a los municipios dentro de cada departamento, también ordenados alfabéticamente.

TABLA 1. Nombre y código de departamentos, municipios, códigos y grupo al que pertenecen

\begin{tabular}{ccccc}
\hline Departamento & $\begin{array}{c}\text { Código } \\
\text { Departamento }\end{array}$ & Municipios & $\begin{array}{c}\text { Código } \\
\text { Municipio }\end{array}$ & Grupo \\
\hline Artigas & 01 & Baltasar Brum & 0101 & Grupo XVII (último) \\
Artigas & 01 & Bella Unión & 0102 & Grupo XVII \\
Artigas & 01 & Tomás Gomensoro & 0103 & Grupo XVII \\
Artigas & 01 & Artigas & 0104 & Grupo XVII \\
Artigas & 01 & Pintadito & 0105 & Grupo XVII \\
Artigas & 01 & Sequeira & 0106 & Grupo VIII \\
Canelones & 02 & Aguas Corrientes & 0201 & Grupo III \\
\hline
\end{tabular}


TABLA 1. Continuación

\begin{tabular}{|c|c|c|c|c|}
\hline Departamento & $\begin{array}{c}\text { Código } \\
\text { Departamento }\end{array}$ & Municipios & $\begin{array}{l}\text { Código } \\
\text { Municipio }\end{array}$ & Grupo \\
\hline Canelones & 02 & Atlántida & 0202 & Grupo II \\
\hline Canelones & 02 & Barros Blancos & 0203 & Grupo I \\
\hline Canelones & 02 & Canelones & 0204 & Grupo III \\
\hline Canelones & 02 & Ciudad de la Costa & 0205 & Grupo I \\
\hline Canelones & 02 & Colonia Nicolich & 0206 & Grupo I \\
\hline Canelones & 02 & Empalme Olmos & 0207 & Grupo II \\
\hline Canelones & 02 & La Floresta & 0208 & Grupo II \\
\hline Canelones & 02 & La Paz & 0209 & Grupo I \\
\hline Canelones & 02 & Las Piedras & 0210 & Grupo I \\
\hline Canelones & 02 & Los Cerrillos & 0211 & Grupo III \\
\hline Canelones & 02 & Migues & 0212 & Grupo II \\
\hline Canelones & 02 & Montes & 0213 & Grupo II \\
\hline Canelones & 02 & Pando & 0214 & Grupo I \\
\hline Canelones & 02 & Parque del Plata & 0215 & Grupo II \\
\hline Canelones & 02 & Paso Carrasco & 0216 & Grupo I \\
\hline Canelones & 02 & Progreso & 0217 & Grupo I \\
\hline Canelones & 02 & Salinas & 0218 & Grupo I \\
\hline Canelones & 02 & San Antonio & 0219 & Grupo III \\
\hline Canelones & 02 & San Bautista & 0220 & Grupo III \\
\hline Canelones & 02 & San Jacinto & 0221 & Grupo II \\
\hline Canelones & 02 & San Ramon & 0222 & Grupo III \\
\hline Canelones & 02 & Santa Lucía & 0223 & Grupo III \\
\hline Canelones & 02 & Santa Rosa & 0224 & Grupo III \\
\hline Canelones & 02 & Sauce & 0225 & Grupo III \\
\hline Canelones & 02 & Soca & 0226 & Grupo II \\
\hline Canelones & 02 & Suárez & 0227 & Grupo I \\
\hline Canelones & 02 & Tala & 0228 & Grupo II \\
\hline Canelones & 02 & Toledo & 0229 & Grupo I \\
\hline Cerro Largo & 03 & Fraile Muerto & 0301 & Grupo X \\
\hline Cerro Largo & 03 & Rio Branco & 0302 & Grupo X \\
\hline Cerro Largo & 03 & Melo & 0303 & Grupo X \\
\hline Cerro Largo & 03 & Isidoro Noblía & 0304 & Grupo X \\
\hline Cerro Largo & 03 & Aceguá & 0305 & Grupo X \\
\hline Cerro Largo & 03 & Hipódromo & 0306 & Grupo X \\
\hline Cerro Largo & 03 & Plácido Rosas & 0307 & Grupo X \\
\hline Colonia & 04 & Carmelo & 0401 & Grupo VI \\
\hline Colonia & 04 & Juan Lacaze & 0402 & Grupo VI \\
\hline Colonia & 04 & Nueva Helvecia & 0403 & Grupo VI \\
\hline Colonia & 04 & Nueva Palmira & 0404 & Grupo VI \\
\hline Colonia & 04 & Rosario & 0405 & Grupo VI \\
\hline Colonia & 04 & Tarariras & 0406 & Grupo VI \\
\hline Colonia & 04 & Colonia del Sacramento & 0407 & Grupo VI \\
\hline Colonia & 04 & Florencio Sánchez & 0408 & Grupo V \\
\hline Colonia & 04 & Ombúes de La Valle & 0409 & Grupo VI \\
\hline Colonia & 04 & Colonia Valdense & 0410 & Grupo VI \\
\hline Colonia & 04 & Colonia de Miguelete & 0411 & Grupo VI \\
\hline Colonia & 04 & Conchillas & 0412 & Grupo VI \\
\hline
\end{tabular}


TABLA 1. Continuación

\begin{tabular}{|c|c|c|c|c|}
\hline Departamento & $\begin{array}{c}\text { Código } \\
\text { Departamento }\end{array}$ & Municipios & $\begin{array}{l}\text { Código } \\
\text { Municipio }\end{array}$ & Grupo \\
\hline Colonia & 04 & La Estanzuela & 0413 & Grupo VI \\
\hline Colonia & 04 & Real de San Carlos & 0414 & Grupo VI \\
\hline Colonia & 04 & La Paz & 0415 & Grupo VI \\
\hline Colonia & 04 & Cufré & 0416 & Grupo VI \\
\hline Colonia & 04 & Artilleros & 0417 & Grupo VI \\
\hline Colonia & 04 & Barker & 0418 & Grupo VI \\
\hline Durazno & 05 & Sarandí del Yí & 0501 & GrupolX \\
\hline Durazno & 05 & Villa del Carmen & 0502 & GrupolX \\
\hline Durazno & 05 & Durazno & 0503 & GrupolX \\
\hline Durazno & 05 & La Paloma & 0504 & GrupolX \\
\hline Durazno & 05 & Centenario & 0505 & Grupo XII \\
\hline Durazno & 05 & Cerro Chato & 0506 & Grupo X \\
\hline Durazno & 05 & Santa Bernardina & 0507 & GrupolX \\
\hline Durazno & 05 & Blanquillo & 0508 & GrupolX \\
\hline Durazno & 05 & Carlos Reyles & 0509 & GrupolX \\
\hline Durazno & 05 & San Jorge & 0510 & GrupolX \\
\hline Flores & 06 & Trinidad & 0601 & Grupo V \\
\hline Flores & 06 & Andresito & 0602 & Entre grupos VIII y IX \\
\hline Florida & 07 & Casupá & 0701 & Grupo III \\
\hline Florida & 07 & Sarandí Grande & 0702 & Grupo IV \\
\hline Florida & 07 & Florida & 0703 & Grupo IV \\
\hline Florida & 07 & Fray Marcos & 0704 & Grupo III \\
\hline Florida & 07 & Veinticinco de Mayo & 0705 & Grupo IV \\
\hline Florida & 07 & Veinticinco de Agosto & 0706 & Grupo VII \\
\hline Florida & 07 & Alejandro Gallinal & 0707 & Entre grupos VII y VIII \\
\hline Florida & 07 & Cardal & 0708 & Grupo IV \\
\hline Florida & 07 & Nico Pérez & 0709 & Grupo X \\
\hline Florida & 07 & Capilla del Sauce & 0710 & GrupolX \\
\hline Florida & 07 & Mendoza Chico & 0711 & Grupo IV \\
\hline Florida & 07 & La Cruz & 0712 & Entre grupos VII y VIII \\
\hline Florida & 07 & Mendoza & 0713 & Grupo IV \\
\hline Florida & 07 & Chamizo & 0714 & Grupo III \\
\hline Florida & 07 & Cerro Chato & 0715 & Grupo X \\
\hline Lavalleja & 08 & José Pedro Varela & 0801 & Grupo X \\
\hline Lavalleja & 08 & Solís de Mataojo & 0802 & Grupo II \\
\hline Lavalleja & 08 & Minas & 0803 & Grupo II \\
\hline Lavalleja & 08 & José Batlle y Ordóñez & 0804 & Grupo X \\
\hline Lavalleja & 08 & Mariscala & 0805 & Grupo X \\
\hline Lavalleja & 08 & Pirarajá & 0806 & Grupo X \\
\hline Lavalleja & 08 & Zapicán & 0807 & Grupo X \\
\hline Lavalleja & 08 & Colón & 0808 & Grupo X \\
\hline Lavalleja & 08 & Illescas & 0809 & Grupo VIII \\
\hline Maldonado & 09 & Aiguá & 0901 & Grupo X \\
\hline Maldonado & 09 & Ciudad de Maldonado & 0902 & Grupo II \\
\hline Maldonado & 09 & Garzón & 0903 & Grupo X \\
\hline Maldonado & 09 & Pan de Azucar & 0904 & Grupo II \\
\hline Maldonado & 09 & Piriápolis & 0905 & Grupo ॥ \\
\hline
\end{tabular}


TABLA 1. Continuación

\begin{tabular}{|c|c|c|c|c|}
\hline Departamento & $\begin{array}{c}\text { Código } \\
\text { Departamento }\end{array}$ & Municipios & $\begin{array}{l}\text { Código } \\
\text { Municipio }\end{array}$ & Grupo \\
\hline Maldonado & 09 & Punta del Este & 0906 & Grupo II \\
\hline Maldonado & 09 & San Carlos & 0907 & Grupo II \\
\hline Maldonado & 09 & Solís Grande & 0908 & Grupo II \\
\hline Montevideo & 10 & & 1001 & Grupo I \\
\hline Montevideo & 10 & & 1002 & Grupo I \\
\hline Montevideo & 10 & & 1003 & Grupo I \\
\hline Montevideo & 10 & & 1004 & Grupo I \\
\hline Montevideo & 10 & & 1005 & Grupo I \\
\hline Montevideo & 10 & & 1006 & Grupo I \\
\hline Montevideo & 10 & & 1007 & Grupo I \\
\hline Montevideo & 10 & & 1008 & Grupo I \\
\hline Paysandú & 11 & Porvenir & 1101 & Grupo XI \\
\hline Paysandú & 11 & Quebracho & 1102 & Grupo XI \\
\hline Paysandú & 11 & Guichón & 1103 & Grupo XI \\
\hline Paysandú & 11 & Paysandú & 1104 & Grupo XI \\
\hline Paysandú & 11 & Nuevo Paysandú & 1105 & Grupo XI \\
\hline Paysandú & 11 & San Felix & 1106 & Grupo XI \\
\hline Paysandú & 11 & Tambores & 1107 & Grupo XIII \\
\hline Paysandú & 11 & Piedras Coloradas & 1108 & Grupo XI \\
\hline Paysandú & 11 & Lorenzo Geyres & 1109 & Grupo XI \\
\hline Paysandú & 11 & Chapicuy & 1110 & Grupo XI \\
\hline Paysandú & 11 & Gallinal & 1111 & Grupo XI \\
\hline Paysandú & 11 & Merinos & 1112 & Grupo XI \\
\hline Río Negro & 12 & Nuevo Berlín & 1201 & Grupo VIII \\
\hline Río Negro & 12 & Young & 1202 & Grupo VIII \\
\hline Río Negro & 12 & Fray Bentos & 1203 & Grupo VIII \\
\hline Río Negro & 12 & San Javier & 1204 & Grupo VIII \\
\hline Rio Negro & 12 & Algorta & 1205 & Entre grupos X y XI \\
\hline Rio Negro & 12 & Grecco & 1206 & Entre grupos X y XI \\
\hline Rivera & 13 & Minas de Corrales & 1301 & Grupo XIV \\
\hline Rivera & 13 & Tranqueras & 1302 & Grupo XIV \\
\hline Rivera & 13 & Vichadero & 1303 & Grupo XIV \\
\hline Rivera & 13 & Rivera & 1304 & Grupo XIV \\
\hline Rivera & 13 & Las Flores & 1305 & Grupo XIV \\
\hline Rivera & 13 & Moirones & 1306 & Grupo XIV \\
\hline Rivera & 13 & Abrojal & 1307 & Grupo XIV \\
\hline Rivera & 13 & Masoller & 1308 & Grupo XV \\
\hline Rocha & 14 & Castillos & 1401 & Grupo X \\
\hline Rocha & 14 & Chuy & 1402 & Grupo X \\
\hline Rocha & 14 & La Paloma & 1403 & Grupo X \\
\hline Rocha & 14 & Lascano & 1404 & Grupo X \\
\hline Rocha & 14 & Rocha & 1405 & Grupo X \\
\hline Rocha & 14 & Cebollatí & 1406 & Grupo X \\
\hline Rocha & 14 & Velázquez & 1407 & Grupo X \\
\hline Rocha & 14 & 18 de Julio & 1408 & Grupo X \\
\hline Rocha & 14 & San Luis al Medio & 1409 & Grupo X \\
\hline Rocha & 14 & La Coronilla & 1410 & Grupo X \\
\hline Salto & 15 & Colonia Lavalleja & 1501 & Grupo XV \\
\hline
\end{tabular}


TABLA 1. Continuación

\begin{tabular}{|c|c|c|c|c|}
\hline Departamento & $\begin{array}{c}\text { Código } \\
\text { Departamento }\end{array}$ & Municipios & $\begin{array}{l}\text { Código } \\
\text { Municipio }\end{array}$ & Grupo \\
\hline Salto & 15 & Mataojo & 1502 & Grupo XV \\
\hline Salto & 15 & Belén & 1503 & Entre grupos XVI y XVII \\
\hline Salto & 15 & Rincón de Valentín & 1504 & Grupo XV \\
\hline Salto & 15 & San Antonio & 1505 & Grupo XVI \\
\hline Salto & 15 & Constitución & 1506 & Grupo XVI \\
\hline Salto & 15 & Salto & 1507 & Grupo XVI \\
\hline Salto & 15 & Colonia de Itapebí & 1508 & Grupo XV \\
\hline San José & 16 & Ciudad del Plata & 1601 & Grupo I \\
\hline San José & 16 & Libertad & 1602 & Grupo VII \\
\hline San José & 16 & San José de Mayo & 1603 & Grupo VII \\
\hline San José & 16 & Rodríguez & 1604 & Grupo VII \\
\hline San José & 16 & Ecilda Paullier & 1605 & Grupo VI \\
\hline San José & 16 & Puntas de Valdez & 1606 & Grupo VII \\
\hline San José & 16 & Rafael Peraza & 1607 & Grupo VII \\
\hline San José & 16 & Ituzaingó & 1608 & Grupo VII \\
\hline San José & 16 & Villa María & 1609 & Grupo VII \\
\hline San José & 16 & Estación González & 1610 & Grupo VII \\
\hline San José & 16 & San Gregorio & 1611 & Grupo VII \\
\hline San José & 16 & Carreta Quemada & 1612 & Grupo VII \\
\hline San José & 16 & Juan Soler & 1613 & Grupo VII \\
\hline San José & 16 & Kiyú & 1614 & Grupo VII \\
\hline San José & 16 & Playa Pascual & 1615 & Grupo I \\
\hline San José & 16 & Cañada Grande & 1616 & Grupo VII \\
\hline Soriano & 17 & Cardona & 1701 & Grupo V \\
\hline Soriano & 17 & Dolores & 1702 & Grupo VI \\
\hline Soriano & 17 & Mercedes & 1703 & Grupo V \\
\hline Soriano & 17 & Palmitas & 1704 & Grupo V \\
\hline Soriano & 17 & José E Rodó & 1705 & Grupo V \\
\hline Soriano & 17 & Villa Soriano & 1706 & Grupo VIII \\
\hline Soriano & 17 & Santa Catalina & 1707 & Grupo V \\
\hline Soriano & 17 & Egaña & 1708 & Grupo V \\
\hline Soriano & 17 & Agraciada & 1709 & Grupo VI \\
\hline Soriano & 17 & Risso & 1710 & Grupo V \\
\hline Soriano & 17 & Sacachispas & 1711 & Grupo VIII \\
\hline Soriano & 17 & Cañada Nieto & 1712 & Grupo VI \\
\hline Tacuarembó & 18 & Tacuarembó & 1801 & Grupo XIII \\
\hline Tacuarembó & 18 & Cañas & 1802 & Grupo XIII \\
\hline Tacuarembó & 18 & Paso del Cerro & 1803 & Grupo XIII \\
\hline Tacuarembó & 18 & Bañado de Rocha & 1804 & Grupo XIII \\
\hline Tacuarembó & 18 & Cuchilla del Ombú & 1805 & Grupo XIII \\
\hline Tacuarembó & 18 & Villa Ansina & 1806 & Grupo XIII \\
\hline Tacuarembó & 18 & El Molino & 1807 & Grupo XIII \\
\hline Tacuarembó & 18 & La Aldea & 1808 & Grupo XIII \\
\hline Tacuarembó & 18 & Zapará & 1809 & Grupo XIII \\
\hline Tacuarembó & 18 & Tacuarembó Chico & 1810 & Grupo XIII \\
\hline Tacuarembó & 18 & Cerro Travieso & 1811 & Grupo XIII \\
\hline Tacuarembó & 18 & Paso del Ceferino & 1812 & Grupo XIII \\
\hline Tacuarembó & 18 & BJD López & 1813 & Grupo XIII \\
\hline
\end{tabular}


TABLA 1. Continuación

\begin{tabular}{|c|c|c|c|c|}
\hline Departamento & $\begin{array}{c}\text { Código } \\
\text { Departamento }\end{array}$ & Municipios & $\begin{array}{c}\text { Código } \\
\text { Municipio }\end{array}$ & Grupo \\
\hline Tacuarembó & 18 & Tambores & 1814 & Grupo XIII \\
\hline Tacuarembó & 18 & Batoví & 1815 & Grupo XIII \\
\hline Tacuarembó & 18 & Curtina & 1816 & Grupo XII \\
\hline Tacuarembó & 18 & Paso de los Novillos & 1817 & Grupo XIII \\
\hline Tacuarembó & 18 & Clara & 1818 & Grupo XIII \\
\hline Tacuarembó & 18 & Paso Hondo & 1819 & Grupo XII \\
\hline Tacuarembó & 18 & Los Cuadrados & 1820 & Grupo XII \\
\hline Tacuarembó & 18 & Carpintería & 1821 & Grupo VIII \\
\hline Tacuarembó & 18 & San Gregorio & 1822 & Grupo XII \\
\hline Tacuarembó & 18 & Achar & 1823 & Grupo XII \\
\hline Tacuarembó & 18 & Peralta & 1824 & Grupo XII \\
\hline Tacuarembó & 18 & Cardozo Grande & 1825 & Grupo VIII \\
\hline Tacuarembó & 18 & Paso de los Toros & 1826 & Grupo XII \\
\hline Tacuarembó & 18 & Pta de Carretas & 1827 & Grupo XIII \\
\hline Tacuarembó & 18 & Pta de Cinco Sauces & 1828 & Grupo XIII \\
\hline Tacuarembó & 18 & Las Toscas & 1829 & Grupo XIII \\
\hline Tacuarembó & 18 & Caraguatá Norte & 1830 & Grupo XIII \\
\hline Tacuarembó & 18 & Rincón de Pereira & 1831 & Grupo XIII \\
\hline Treinta y Tres & 19 & Santa Clara de Olimar & 1901 & Grupo X \\
\hline Treinta y Tres & 19 & Vergara & 1902 & Grupo X \\
\hline Treinta y Tres & 19 & Treinta y Tres & 1903 & Grupo X \\
\hline Treinta y Tres & 19 & Cerro Chato & 1904 & Grupo X \\
\hline Treinta y Tres & 19 & Villa Sara & 1905 & Grupo X \\
\hline Treinta y Tres & 19 & Estación Rincón & 1906 & Grupo X \\
\hline
\end{tabular}

Para el análisis se utilizó el programa dBXL desarrollado por WordTechSystems, Inc., disponible en www.vetusware.com/download/dBXL\%201.2/?id=3978. Con él se elaboraron bases de datos de los apellidos en cada municipio y con estas se hicieron los cálculos que se describen a continuación.

\section{Distancias isonímicas}

Se calcularon las distancias isonímicas de Lasker (Lasker, 1977; Rodríguez-Larralde et al., 1998), euclídea (Cavalli-Sforza y Edwards, 1967) y de Nei (Nei, 1973) entre los 221 municipios. A la isonimia dentro del municipio $\mathrm{J}$ la llamaremos $\mathrm{I}_{\mathrm{j} j}=\sum_{\mathrm{k}} \mathrm{p}_{\mathrm{kj}}{ }^{2}$, donde $\mathrm{p}_{\mathrm{kj}}$ es la frecuencia relativa del apellido $\mathrm{k}$ en ese municipio, y la isonimia entre municipios I y J (equivalente al coeficiente de parentesco de Lasker mencionado anteriormente) fue estimada como $\mathrm{I}=\sum_{\mathrm{k}} \mathrm{p}_{\mathrm{ki}} \mathrm{p}_{\mathrm{kj}}$, donde $\mathrm{p}_{\mathrm{ki}}$ y $\mathrm{p}_{\mathrm{kj}}$ son las frecuencias relativas del apellido $\mathrm{k}$ en esos dos municipios, y la suma considera todos los apellidos. La distancia de Lasker se define como $L=-\log \left(I_{i j}\right)$. La distancia euclídea viene dada por $E=\sqrt{ }\left(1-\sum_{k} \sqrt{ } p_{k i} p_{k j}\right)$, y la de Nei por $N d=-\log \left(I_{i j} / \sqrt{ }\left(I_{i i j j} I\right)\right)$, todas calculadas con el programa DISEUNE1 escrito en lenguaje Fortran por ARL.

Se estimó la correlación entre las distintas distancias isonímicas y geográficas mediante la prueba de Mantel (Mantel, 1967; Smouse, Long y Sokal, 1986) con 1000 permutaciones, utilizando el programa Bootmat escrito en lenguaje Fortran por ARL.

La representación gráfica de las matrices de distancia se hizo mediante dendrogramas con los métodos de neighbor-joining (Saitou y Nei, 1987) y UPGMA (Sokal y Michener, 1958) 
utilizando el paquete PHYLIP (Felsenstein, 1989; 1993) disponible en: http://Mobile.pasteur. $\mathrm{fr} / \mathrm{cgi}$-bin/portal.py. La identificación de los agrupamientos en el dendrograma se hizo trazando una línea perpendicular a su base, de forma relativamente arbitraria para separar los agrupamientos de tamaño intermedio (Fig. 1). Los grupos son mutuamente excluyentes y se identifican con números romanos en el orden que ingresan al dendrograma.

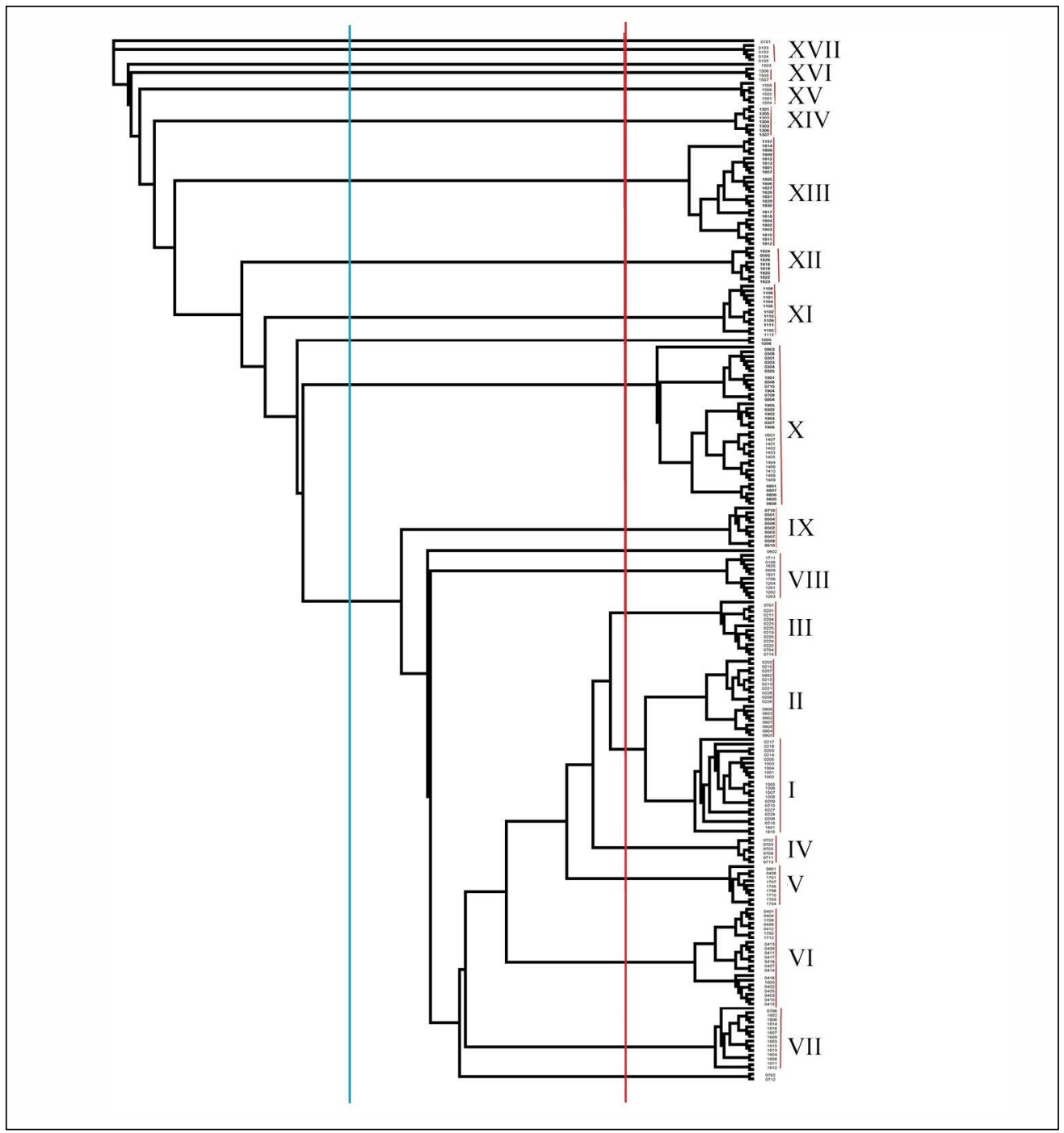

FIGURA 1. Dendrograma representando la matriz de distancias euclídeas entre municipios de Uruguay, en el cual se observa la estructura de los agrupamientos.

\section{RESULTADOS}

El uso de varias distancias permitió comparar sus resultados. La correlación entre distancia geográfica y distancias isonímicas en base a 24.310 pares de datos fue coherente con los distintos métodos, con ligeras diferencias: mayor para la distancia euclídea $(r=0,253$ $\pm 0,033)$, intermedia para la de Lasker $(r=0,18 \pm 0,029)$ y menor para la de Nei $(r=0,17 \pm 0,03)$, todas estadísticamente significativas $(p<0,001)$. Puesto que los resultados obtenidos con las distancias fueron, en grandes términos, similares, se presentan en adelante los obtenidos con base en la distancia euclídea ya que fue la que mostró mayor correlación con la distancia geográfica.

La Figura 1 muestra el dendrograma obtenido con las distancias euclídeas y el método de neighbor-joining, donde se observa la estructura general de los agrupamientos; esta 
figura se complementa con la Tabla 1, donde se presenta la identificación de los grupos a los que pertenece cada municipio. Se observan 17 grupos mutuamente excluyentes que se identifican con números romanos en el orden que van ingresando al dendrograma (línea roja), que pudieran reducirse a 9 agrupamientos (línea azul), quedando en este último caso unidos prácticamente todos los municipios al sur del río Negro, como se detalla más adelante. Distancias pequeñas entre municipios indican que entre ellos la distribución de los apellidos es similar, lo cual puede deberse a que los individuos fundadores portaban los mismos apellidos por azar, por estar emparentados o porque las migraciones entre ellos son abundantes. La representación aproximada de estos grupos sobre el mapa geográfico del Uruguay se muestra con líneas punteadas en la Figura 2.

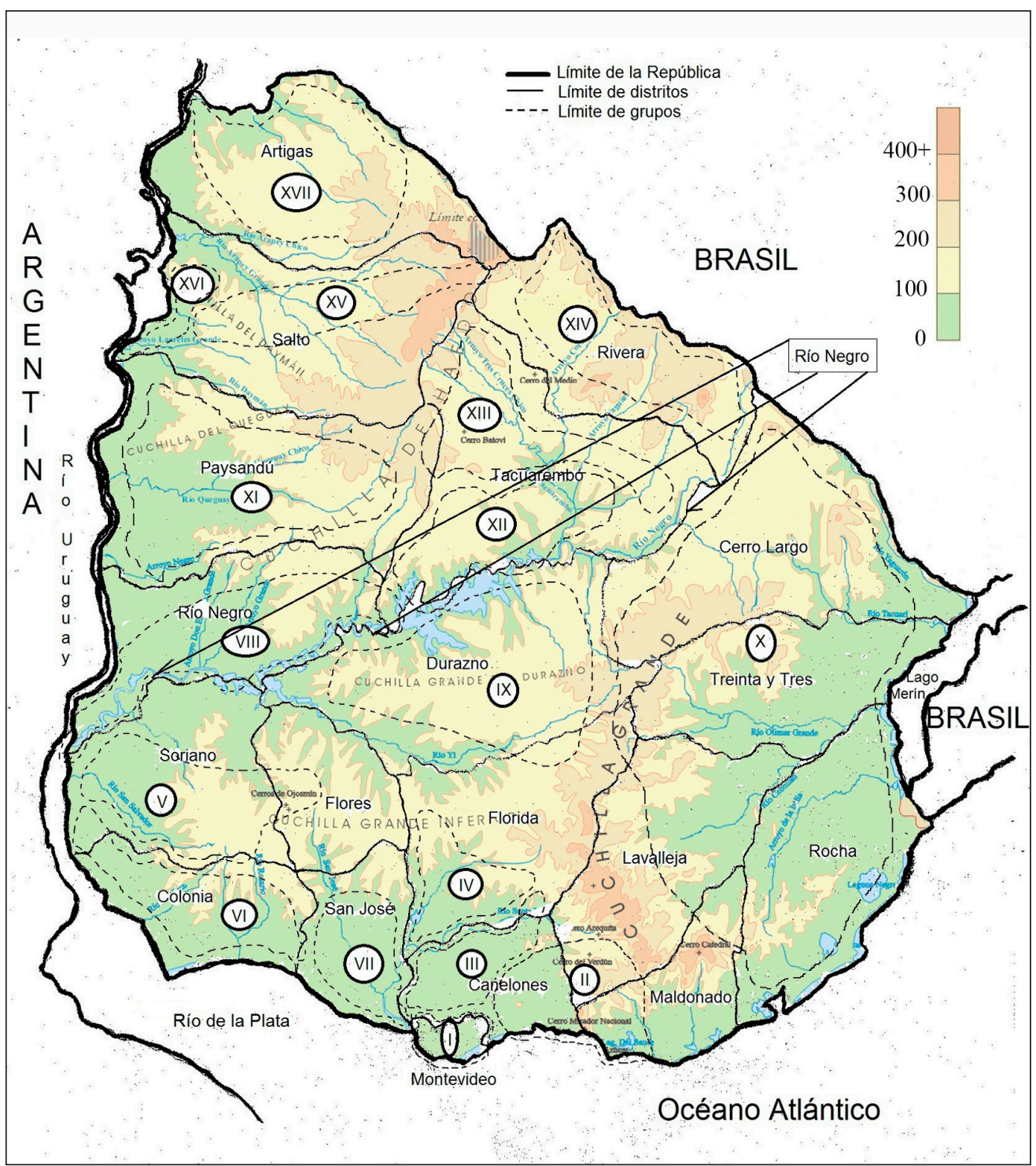

FIGURA 2. Mapa de la República del Uruguay mostrando departamentos y principales accidentes geográficos mencionados en el texto, con la identificación de los grupos representados en la figura 1.

Se designaron como grupos I y II aquéllos a los que se van agregando los otros grupos, a distancias cada vez mayores, llegando a los últimos grupos que incluyen los municipios con la distribución de apellidos que más difiere del resto. Llama la atención que, en líneas generales, los grupos están formados por municipios de un mismo departamento o pertenecientes a departamentos diferentes pero muy cercanos geográficamente. 
El corazón del dendrograma está en los grupos I, II y III. El grupo I está formado por municipios de Montevideo (código 10), a los que se van agregando municipios de Canelones (código 02) y, por último, el par 1601 y 1615 (Ciudad del Plata y Playa Pascual) del departamento San José, limítrofe con Montevideo y parte del área metropolitana de esta ciudad. El grupo Il consiste de dos subgrupos: en uno de ellos todos los municipios son de Canelones (código 02), a excepción de 0802 (Lavalleja), en el otro, todos son de Maldonado (código 09) menos 0803 (también de Lavalleja); el departamento de Lavalleja es limítrofe tanto con Canelones como con Maldonado y los municipios mencionados están en esa zona limítrofe. Muy cerca a los dos anteriores, ingresa el grupo III, formado por municipios de Canelones (código 02) y tres municipios de Florida (código 07), muy cerca de la frontera con Canelones.

Paulatinamente comenzamos a alejarnos del centro capitalino. El grupo IV está formado por municipios de Florida (código 07) y luego el V, formado principalmente por municipios de Soriano. El grupo VI consiste en municipios del departamento de Colonia (código 04), de Soriano, cerca de la frontera con Colonia (1709, 1702 y 1712) y el municipio 1605, del departamento de San José, también en la frontera con Colonia. En el grupo VII todos son municipios de San José (código 16), menos uno de los últimos en entrar en este grupo, 0706, situado en el vértice sur del departamento de Florida, limítrofe con San José. El grupo VIII tiene dos subgrupos: el de la izquierda formado por municipios del departamento Río Negro (código 12), el primero situado al norte del país (tomando como límite norte-sur el cauce del río Negro) y el de la derecha, muy heterogéneo, con municipios de Tacuarembó, Lavalleja, Artigas y Soriano. Llama la atención en este último agrupamiento que dos departamentos corresponden al norte y dos al sur del país (considerando al río Negro divisor de estas dos regiones, como ya se ha mencionado) estando los cuatro municipios lejanos geográficamente entre sí. El siguiente grupo es el IX, todos de Durazno (código 05) menos Capilla del Sauce (0710), en Florida, limítrofe con Durazno. Todos estos municipios (marcados en el dendrograma bajo la línea azul) están situados al sur del río Negro, exceptuando el heterogéneo grupo VIII (Fig. 1). A partir del grupo IX se observa un incremento en las distancias isonímicas y un nuevo arreglo poblacional. Este grupo, relativamente heterogéneo, está formado por municipios del norte y el oeste y también quedaría integrado a los municipios del sur (Fig. 1).

Se incorpora después el grupo $\mathrm{X}$, con municipios de los departamentos de Lavalleja, Rocha, Cerro Largo y Treinta y Tres, ubicados hacia el este de Uruguay y al sur del río Negro a excepción de Cerro Largo, lo cual llama la atención puesto que se esperaría que este grupo se vinculara, por su ubicación geográfica, más cercano al núcleo de los agrupamientos. Sin embargo, se separa claramente (Fig. 1).

En cuanto a los conjuntos siguientes (todos en el norte del país), el XI está formado por municipios de Paysandú, mientras que el XII incluye municipios de Tacuarembó, en el norte y este, a excepción de uno (0505), en la ribera sur del río Negro pero en el área de influencia de la ciudad de Paso de los Toros, en la ribera norte del río Negro y separada por un puente del municipio mencionado y donde antes había un vado. Todos los municipios del XIII también son de Tacuarembó, a excepción del 1107, en la localidad de Tambores (Paysandú), en la frontera con Tacuarembó y en el área de influencia de la capital de este último departamento. A continuación aparecen los grupos XIV, XV, XVI y XVII, ubicados hacia el norte del país. El grupo XIV, formado por municipios de Rivera (código 13), en el noreste y el grupo XV en el noroeste, todos de Salto (15), menos 1308, en frontera con Salto. Los del XVI también son de Salto y luego se incorpora al dendrograma el municipio 1503, Belén, en la frontera con Artigas y el único que aparece solo. Posteriormente se integra el grupo XVII, que inclu- 
ye municipios de Artigas (01), el departamento más al norte y más distante de los demás, siendo el municipio 0101, Baltasar Brum, el último en ingresar al dendrograma. Todos estos agrupamientos están claramente definidos y separados de los señalados como I a IX (Fig. 1).

Con relación a los apellidos, si bien no fue el objetivo de este estudio, puede señalarse que a nivel general, la gran mayoría es de origen español. Los agrupamientos del norte y del este (X a XVII) presentan mayor cantidad de apellidos portugueses. Solo se identificaron 2 apellidos de origen indígena, por lo cual no fue posible analizarlos a nivel espacial, y no se identificaron apellidos que puedan claramente relacionarse a población de origen africano.

\section{DISCUSIÓN}

En este trabajo se calculan las distancias isonímicas para evaluar la distribución geográfica de los apellidos a lo largo del país. A partir de estas distancias se generan agrupamientos, que pueden observarse en los dendrogramas, proporcionando una aproximación a la estructura jerárquica de la distribución espacial de su población. Se verifica el aislamiento por distancia de acuerdo al modelo de Malécot (1955), un dato que ya fue revelado en estudios previos a nivel departamental (Carrieri et al., 2020). La mayor correlación entre distancias geográficas e isonímicas fue con la distancia euclídea $(r=0,253, p<0,001)$. De todos modos, esta asociación es menor a la observada en el análisis de Carrieri y colaboradores ya citado, quienes obtuvieron, para la distancia de Nei calculada a nivel departamental, un valor de correlación de $r=0,57(p<0,001)$. Con la misma distancia de Nei, pero analizada aquí entre municipios, fue de $r=0,17$ ( $p<0,001)$. A pesar de que en ambos trabajos las correlaciones son altamente significativas, la diferencia en magnitud puede explicarse porque en el trabajo de Carrieri y colaboradores el número de comparaciones era reducido. La estimación fue hecha con 171 comparaciones (19 departamentos tomados 2 a 2), mientras que en el presente trabajo fue hecha con 24.310 comparaciones (221 municipios tomados 2 a 2).

El análisis con un mayor detalle poblacional sugiere la influencia de una multiplicidad de factores geográficos y demográficos que actuaron en esta jerarquización. Esta puede interpretarse también a partir de la primera ley de la geografía, o principio de autocorrelación espacial formulado por Tobler (1970) que refuerza el modelo Malécot (1955). El principio de Tobler plantea que los objetos están relacionados entre sí, pero aquéllos más próximos en el espacio tienen una relación mayor que los distantes. Fue aplicado recientemente por Shi et al. (2019a) y Shi, Li, Wang, Chen y Stanley (2019b) para interpretar la estructura jerárquica de las poblaciones chinas establecida a partir de distancias isonímicas.

El total de municipios del Uruguay fue agrupado independientemente de los departamentos a los cuales pertenecían. El corazón o núcleo de agrupamientos iniciales (I, II y III) se interpreta, por una parte, como la consecuencia de la migración de las zonas rurales del Uruguay hacia la capital y zonas aledañas, fenómeno típico en los países latinoamericanos que muestran una acentuada tendencia a la urbanización y concentración de la población en determinadas ciudades de gran tamaño (Farías, 1989). La capital y sus alrededores reciben población de todo el país, pero es de esperarse que reciban una mayor proporción de los departamentos más cercanos. A esto se agrega otro factor de importancia, el hecho que tanto la capital como las zonas aledañas recibieron el mayor flujo de inmigrantes europeos y de Próximo Oriente, que entraban por el puerto de Montevideo, único en recibir migrantes de ultramar. El rápido crecimiento de la población durante el siglo XIX se puede ver en censos y estimaciones: por ejemplo, en 1873, la población total ascendía a 450.000 habitantes, lo que indica que entre 1800 y 1873 se había multiplicado por 14,5, fundamentalmente a expensas de los ingresos por el puerto de Montevideo (Barrán y Nahum, 1979). 
Estos inmigrantes provenían de diversos orígenes: españoles de distintas regiones, franceses, vascos (franceses y españoles), italianos, que llegaban en diversas oleadas, radicándose en las ciudades o en los campos fundamentalmente al sur del río Negro (Pi y Vidart, 1969). La posición de los municipios de Montevideo y departamentos limítrofes (Canelones, San José) o cercanos (Maldonado, parte de Lavalleja y de Florida) refleja las características de esa inmigración, formando el núcleo del dendrograma a partir del cual se van incorporando otros grupos de acuerdo a las distancias isonímicas estimadas. Con escasas excepciones, el orden en el que se agregan los demás grupos también está relacionado con la ubicación geográfica de los departamentos, reforzando así el concepto de Tobler.

Al igual que para los tres primeros agrupamientos, los siguientes (IV al X, aunque con la excepción de parte del XVIII) también parecen estar fuertemente condicionados por los antecedentes demográficos del Uruguay y su relativa cercanía a la capital. A modo de ejemplo podemos analizar tres grupos poblacionales llegados mayoritariamente en el siglo XIX: italianos, vascos y canarios, grupos que si bien tienen antecedentes previos de inmigración -en particular los últimos, quienes participaron en la fundación de Montevideo- hubo cuantitativamente mayor afluencia en el siglo XIX. Su radicación e inserción a la sociedad uruguaya dependen de condiciones de su origen: los vascos fundamentalmente se dispersaron en las estancias relacionadas con la cría de ovinos y bovinos o la lechería, con paisajes similares a las de su lugar de origen, en las regiones centro-sur, litoral y serrana del sur de país (Marenales y Luzuriaga,1990), mientras que otros se instalaron en los centros urbanos también en el sur, dedicados al pequeño comercio u otras actividades y muchas veces obligados a reembolsar con su trabajo el costo del viaje (Pi y Vidart, 1969). Los migrantes de Islas Canarias se radicaron preferentemente en quintas del departamento de Canelones, desde donde proveían a la ciudad de Montevideo (Martínez Díaz, 1985). Por último, los italianos, se establecieron fundamentalmente en regiones agrícolas del sur del país, aunque también en el litoral noroeste; según su origen se dedicaron a la agricultura o a la ganadería, o al trabajo independiente en centros urbanos (Bresciano, 2010). La inmigración europea de inicios del siglo XX, constituida sobre todo por españoles e italianos, también se radica mayoritariamente en Montevideo; a ellos se suman en las décadas siguientes los ingresos de migrantes provenientes del Medio Oriente y de Europa Oriental (Pellegrino, Cabella, Paredes, Pollero y Varela, 2008). Aún en la actualidad y de acuerdo a datos del Censo de 2011, en los departamentos fronterizos con Brasil (Rivera, Cerro Largo y Artigas) se observa un claro predominio de los extranjeros nacidos en ese país, mientras que en los departamentos fronterizos con Argentina (Colonia, Soriano, Río Negro y Paysandú) se incrementa notoriamente el porcentaje de población nacida en Argentina (OIM, 2011). Por otra parte, Ilama la atención que el departamento de Colonia, característico por tener enclaves de inmigrantes suizos, alemanes (municipio de Nueva Helvecia) y valdenses (Colonia Valdense) (Pi y Vidart, 1969), cuyos municipios integran el grupo VI, no se separe de los agrupamientos del sur del país, lo cual podría deberse a procesos recientes de migración interna.

En los agrupamientos I a IX predominan los apellidos españoles, aunque se advierten múltiples orígenes coherentemente con las distintas oleadas migratorias mencionadas. Sin embargo, no fue objetivo de este trabajo detallar la estructura poblacional de acuerdo al origen y características de los apellidos, lo cual será objeto de un futuro estudio. Más datos sobre los apellidos pueden encontrarse en Carrieri y colaboradores (Carrieri et al., 2020).

Más compleja es la consideración de los agrupamientos del norte, que reciben algunos de los migrantes ya mencionados -ingresados por Montevideo- pero también y fundamentalmente, pobladores desde Brasil, ya desde el siglo XVII. Entre 1816 y 1828 ocurre la ocupación luso-brasileña del actual territorio uruguayo, período en el que se reparten tierras a 
oficiales y soldados de ese origen. Pero ya desde el siglo XVII y también posteriormente a la ocupación mencionada, familias portuguesas o brasileñas compran tierras en la región, muchas veces escapando de las luchas en el vecino estado de Río Grande do Sul (Brasil). Paralelamente, también ingresan al territorio africanos y sus descendientes que huyen de la esclavitud luego de abolida ésta en nuestro país en la década de 1840 y hasta 1888, cuando Brasil hace lo propio. Tal era la situación del norte y nordeste del Uruguay, que en 1845 el diputado brasileño Silva Ferraz señalaba ante el Parlamento de su país que, al pasar al otro lado del río Yaguarón, ya en territorio uruguayo, tanto el idioma como las costumbres, la moneda, e incluso la tierra, eran brasileños (Acevedo, 1933). Con relación al departamento de Tacuarembó, indica González Mieres (1968) que hasta la tercera década del siglo XX el lenguaje de los pobladores era híbrido, mezcla de castellano y portugués; recién en la década de 1930 comienza la recuperación del español a través de las escuelas. Paralelamente a la afluencia de brasileños en el norte y noreste, se observa una presencia importante de argentinos en el litoral del río Uruguay (Pellegrino, Cabella, Paredes, Pollero y Varela, 2008). En estos agrupamientos, si bien continúan predominando los apellidos españoles, la cantidad de apellidos de origen portugués aumenta considerablemente.

A los factores demográficos se agrega un factor geográfico que parece haber actuado como barrera determinante: el rio Negro, que atraviesa el país de este a oeste a lo largo de un recorrido de $750 \mathrm{~km}$ que solo presenta actualmente 5 puentes, correspondientes a rutas principales. La mayoría de los agrupamientos respeta esta barrera, estando los agrupamientos I al X del lado sur y del XI al XVII del lado norte. Como excepciones encontramos que el agrupamiento XII incluye una población (Centenario) en el departamento de Durazno, al sur. Este resultado es fácilmente explicable por la existencia histórica de un paso y desde 1930, de un puente, cuya construcción llevó a la radicación de obreros en la localidad mencionada. Otras dos excepciones son el agrupamiento $X$, que incluye varios departamentos del este, al sur del río Negro pero con gran aporte luso-brasileño (que será tratado en profundidad más adelante) y el agrupamiento VIII, el más complejo de todos puesto que incluye municipios del departamento de Río Negro, en el litoral centro-norte, pero también algunos municipios del sur (Lavalleja y Soriano, en conjunto con municipios norteños de Artigas y Tacuarembó). La división de países o regiones por accidentes hidrográficos y orográficos no es una novedad, pueden encontrarse diversos ejemplos de ríos como reguladores de la movilidad humana y, por lo tanto, de la distribución de apellidos. Por ejemplo, la relación de las parroquias de la región del delta del río Ebro en España, los ríos Yangtse y Amarillo en China y el rio Bermejo en la frontera argentino-boliviana (Dipierri, Alfaro, Rodríguez-Larralde y Ramallo, 2016; Esparza, García Moro y Hernández 2006; Shi et al., 2019a, Shi et al., 2019b).

Aunque la orografía de Uruguay se caracteriza por su homogeneidad y con características de penillanura, con alturas que no superan los 500 m.s.n.m., llama la atención la disposición de los agrupamientos en relación a las cuchillas que constituyen las formas de relieve más frecuentes en el territorio uruguayo. Hacia el norte de Uruguay se destaca la cuchilla de Haedo que separa a los agrupamientos XVII, XV, XVI, y XI hacia el oeste de los agrupamientos XIV, XIII y XII hacia el este. Hacia el sureste del país el extenso agrupamiento X, que incluye a 36 municipalidades, se separa de los restantes por la cuchilla Grande. El agrupamiento IX se asienta exclusivamente sobre la cuchilla Grande de Durazno y la cuchilla Grande Interior separa a los agrupamientos situados sobre el litoral platense (agrupamientos I a VII) del resto de los conjuntos. Dada la poca altura de estas cuchillas, podría relacionarse estas separaciones no solo al factor orográfico en sí mismo, sino a la demarcación de tierras ocurridas históricamente y que se apoyaban en elementos naturales: por ejemplo, el Reglamento de 
Tierras de 1815 de José Gervasio Artigas autorizaba al alcalde provincial tanto a distribuir tierras para incentivar el poblamiento del país, así como a repartir entre los revolucionarios orientales que habían participado en las luchas por la independencia aquellas parcelas tomadas a extranjero. En el artículo tercero se mencionaban las cinco regiones en la que se separaba al país, todas ellas divididas por accidentes hidrográficos: entre los ríos Uruguay y Negro, Negro y Yí, entre el Santa Lucía a la costa del mar, y del Yi hasta el Santa Lucía, límites que aún separan varios de los departamentos del sur del país (Padrón Favre, 2011). En el mismo Reglamento, el artículo 16 menciona que se demarcarían terrenos de legua y media de frente y dos de fondo, pudiendo variar según el terreno, con aguadas y en lo posible con "linderos fijos" que evitaran posibles desavenencias entre vecinos (Artigas, 1815). Debe entenderse que estos linderos fijos refieren fundamentalmente a factores orográficos e hidrográficos, ya que la demarcación mediante otros métodos, como, por ejemplo el alambrado de los campos, recién comienza en 1872.

El agrupamiento poblacional y la sectorización del espacio nacional alcanzada en este trabajo con base a los apellidos constituye un aporte a los estudios de regionalización y territorialización del Uruguay. Si bien, de acuerdo a Bourdieu (1985), las regiones delimitadas según diversos parámetros como lengua, hábitat, u otros factores socioeconómicos nunca coinciden totalmente entre sí, es de interés analizar algunas semejanzas con otras regionalizaciones. Si se compara el mapa de agrupamientos alcanzado en este trabajo con base a isonimia, con los analizados por Yagüe y Díaz-Puente (2008) en su revisión sobre descentralización, solo se equipara parcialmente con la propuesta de Quagliotti y Chamorro (1977), quienes definen 5 zonas y un área metropolitana en torno a los ejes de desarrollo, en parte semejante a las regiones isonímicas. Esto permite hipotetizar que probablemente el agrupamiento isonímico de las poblaciones uruguayas se encuentre también condicionado por factores socioeconómicos ligados a las desigualdades sociales y territoriales del Uruguay (Veiga, 2015). Además, es interesante notar cómo las regiones isonímicas también coinciden parcialmente con las basadas en índices de desarrollo, como el índice de desarrollo humano y el ingreso promedio de los hogares (Bittencourt, 2009), si bien las regiones basadas en estos valores incluyen más de un área isonímica. Las siete regiones planteadas son las siguientes: 1- Montevideo, 2- Canelones, 3- Centro (Flores, Durazno y Florida), 4Suroeste (San José, Colonia y Soriano), 5- Litoral (Salto, Paysandú y Río Negro), 6- Norte (Artigas, Tacuarembó, Rivera y Cerro Largo) y 7- Este (Maldonado, Lavalleja, Rocha y Treinta y Tres), áreas que son retomadas en un estudio sobre regionalización cultural (Arocena y Gamboa, 2011). Todos los indicadores separan a Montevideo, seguido por Centro -ambas con mejores índices de desarrollo- del resto del país, mientras que los peores índices se encuentran en el norte-noreste y Canelones, este último con resultados discutibles según los propios autores ya que está afectado por el hecho que muchos de estos habitantes trabajan en Montevideo, lo cual modifica el producto interno departamental. La relación con los agrupamientos isonímicos tiene algunos aspectos a destacar: en primer lugar, el caso de Cerro Largo. Si bien geográficamente se ubica en el este, se diferencia por su peor situación socio-económica y aparece relacionado a las localidades del norte-noreste por su estado de desarrollo e ingresos. Los municipios de Cerro Largo se ubican al sur del río Negro y corresponden al agrupamiento $\mathrm{X}$, al igual que Treinta y Tres y Rocha, pero se integran al dendrograma separadamente de los del sur, entremezclado con los primeros del norte. Es posible que aquí la influencia luso-brasileña haya sido más fuerte que la barrera geográfica del río Negro en sí, ya que tiene escaso caudal en esa región, más fuerte aún que las distancias geográficas lineales.

Cabe destacar que la diferencia encontrada en nuestro estudio entre el norte y sur del 
Revista

Argentina de

Antropología Biológica

\section{AGRADECIMIENTOS}

Al Sr. Claudio Martínez Povedo por su colaboración en la obtención de los padrones de Tacuarembó. A la Ay. Arq. Miranda Hidalgo por la confección de la figura 2.
Río Negro, aunque con excepciones, se observa claramente en los distintos indicadores, tanto de desarrollo como culturales. Señala Padrón Favre (2011), tomando las siete regiones mencionadas, que las cuatro regiones meridionales (Montevideo, Canelones, Sudoeste y Este) tuvieron una configuración socio-económica y político-administrativa más temprana, en su mayor parte, con capitales de la época colonial, mientras que al norte del río Negro solamente dos capitales son de ese período, una en el noreste y otra en el oeste. La dicotomía norte-sur continuó en el período revolucionario y posteriormente, aún el más exitoso litoral norte fue subordinado al poder central.

Por último, hay otro elemento relativamente vinculado que puede considerarse en la regionalización encontrada por isonimia: la ancestría. En el Censo Nacional de 2011 (INE, 2011), un promedio de 4,9\% de la población reconoció poseer ancestros indígenas y un 2,5 declaró la indígena como su ancestría principal. Estos valores cambian por departamentos y regiones: los valores máximos se encuentran en Tacuarembó (8\%) y Salto (6\%), seguidos de Artigas y Rivera (5\%), todos en el norte del país (agrupamientos isonímicos XIII al XVII, que están entre los más lejanos al núcleo de la capital) y los valores mínimos se encuentran en la región sur y litoral sur (por ejemplo en Colonia, Flores y Florida, donde se encuentran los agrupamientos IV a VI, el valor de ancestría indígena es de entre 1 y $2 \%$ ). También parte del grupo $X$, en el este del país, presenta valores de ascendencia indígena algo mayores al promedio (5\%), lo cual avala la posición en el dendrograma, más relacionado a municipios del norte. Por otra parte, el departamento de Río Negro, si bien forma parte del litoral norte (agrupamiento VIII), tiene bajos valores de reconocimiento de ascendencia indígena (3\%), lo cual sería coherente con la escasa distancia a los municipios del sur. Montevideo y alrededores (grupos I a III) tienen situaciones intermedias, cercanas a la media (4\%), pero podría explicarse por las migraciones recientes hacia la capital (INE, 2011).El panorama con relación a la ascendencia africana es similar, con valores máximos en Rivera y Artigas (17\%, agrupamientos XIV y XVII), seguido por Cerro Largo (11\%, grupo X), Salto y Tacuarembó (10\%, grupos XII, XIII, XV y XVI). Colonia, Soriano y Florida tienen los menores valores (3\%, agrupamientos IV a VI). En conjunto, además de la separación sur-norte, se puede observar la separación este-oeste, con valores menores en el sudoeste. Nuevamente, Montevideo y alrededores (agrupamientos I a III) tiene un aporte intermedio, aunque más similar al del norte (9\%) (INE, 2011).

Si bien los datos de reconocimiento de ascendientes indígenas o africanos son discutibles y no necesariamente reflejan el origen genético de la población, puesto que fueron brindados por los habitantes del país al momento del Censo, se ha visto que el aporte indígena en particular está claramente subestimado (Sans, 2009). Los datos genéticos que poseemos respaldan los datos censales en cuanto a la distribución espacial, con mayores valores de ancestría indígena en Tacuarembó y Bella Unión (Artigas) (62-64\% por vía materna) y menores en el sur (21\% por vía materna), mientras que los mayores valores de ancestría africana se obtuvieron en Tacuarembó, Cerro Largo y también Montevideo (17\%, 21\% y $11 \%$ respectivamente) (Salzano y Sans, 2014). Sería deseable tener datos genéticos de más regiones y con mayor detalle dentro de éstas para profundizar el análisis.

\section{CONCLUSIONES}

El análisis de los apellidos permitió identificar agrupamientos poblacionales que muestran un efecto importante de las distancias geográficas, los factores histórico-demográficos y los accidentes geográficos, en su relación con la actividad socio-política-administrativa en la concentración de focos poblacionales en la República del Uruguay. Grosso modo, se 
identifica un gran foco poblacional al sur del río Negro, que aumenta sus distancias tomando como centro la ciudad capital, mientras que los municipios del norte y nordeste están agregados por distancia geográfica pero sin constituir un agrupamiento independiente. Un segundo eje, menos determinante, separa los municipios de la región fronteriza con Brasil, de aquellos del litoral oeste.

A futuro, análisis más detallados podrían intentar correlacionar los agrupamientos isonímicos identificados con otros factores, como socioeconómicos o genéticos.

\section{LITERATURA CITADA}

Acevedo, E (1933). Anales históricos del Uruguay, Montevideo: Barreiro y Ramos.

Arocena, F. y Gamboa, M. (2011). Marco conceptual e hipótesis de trabajo para la regionalización cultural. En: F. Arocena (Ed.). Regionalización cultural del Uruguay (pp. 17-44), Montevideo: Universidad de la República.

Artigas, J. G. (1815). Reglamento Provisorio de la Provincia Oriental para el Fomento de la Campaña y Seguridad de sus hacendados. Consultado en: https://www.biblioteca.org.ar/libros/158184.pdf

Barrai, I., Scapoli, C., Mamolini, E. y Rodríguez-Larralde, A. (1999). Isonymy and isolation by distance in Italy. Human Biology, 71, 947-961. doi:10.1353/hub.2002.0016

Barrai, I., Rodríguez-Larralde, A., Mamolini, E., Manni, F. y Scapoli, C. (2001). Isonymy structure of USA population. American Journal of Physical Anthropology, 114, 109-123. doi:10.1002/10968644(200102).

Barrán, J.P. y Nahum, B. (1979). Batlle, los estancieros y el Imperio Británico, Montevideo: Banda Oriental.

Bittencourt, G. (2009). Estrategia Uruguay Tercer Siglo. Aspectos productivos, Montevideo: Oficina de Planeamiento y Presupuesto.

Bresciano, J. (2010). La inmigración italiana al Uruguay en perspectiva historiográfica, en: Rita C.M (Ed.): Un paesechi cambia. Saggi antropologici sull'Uruguay tra memoria e attualità (pp. 111-136), Roma: Centro de Informazione e Stampa Universitaria.

Bourdieu, P. (1985). ¿Qué significa hablar? Economía de los intercambios lingüísticos. Madrid: Akal.

Carrieri, A., Sans, M., Dipierri, J.E., Alfaro, E.L.A., Mamolini, E., Sandri, M.y Barrai, I. (2020). The structure and migration patterns of the population of Uruguay through isonymy. Journal of Biosocial Science, 52(2), 300-314. https://doi.org/10.1017/S0021932019000476

Cavalli-Sforza, L. y Edwards, A. W. F. (1967). Phylogenetic analysis: Models and estimation procedures. American Journal of Human Genetics, 19, 233-257. doi:10.1111/j.1558-5646.1967.tb03411.x

Colantonio, S. E., Lasker, G. W., Kaplan, B. A. y Fuster, V. (2003). Use of surname models in human population biology: a review of recent developments. Human Biology, 75, 785-807. doi:10.1353/ hub.2004.0004

Crow, J. F. y Mange, A.(1965). Measurements of inbreeding from the frequency of marriages between persons of the same surnames. Eugenics Quarterly, 12, 199-203.

Dipierri, J. E., Alfaro, E. L., Scapoli, C., Mamolini, E., Rodríguez- Larralde, A. y Barrai, I. (2005). Surnames in Argentina. A population study through isonymy. American Journal of Physical Anthropology, 128, 199-209. doi: 10.1017/S0021932016000444

Dipierri, J. E., Alfaro, E. L., Rodríguez-Larralde, A. y Ramallo, V. (2016) Isonymic relations in the BoliviaArgentina border. Human Biology, 88, 191-200. doi:10.13110/humanbiology.88.3.0191

Esparza, M., García-Moro, C., Hernández, M. (2006). Genetic relationships between Parishes in the Ebro Delta Region (Spain) as estimated by migration matrix and surnames. Human Biology, 78, 647-662. doi:10.1353/hub.2007.0010

Farías, D.O. (1989). Las metrópolis latinoamericanas y sus tendencias. Revista Geográfica, 110, 21-27. 
Felsenstein, J. (1989). PHYLIP - Phylogeny Inference Package (version 3.2). Cladistics, 5, 164-166.

Felsenstein, J. (1993). PHYLIP - Phylogeny Inference Package (version 3.5c). Distributed by the author. Seattle: University of Washington, Department of Genetics.

González Mieres, L. (1968). El “Homo tacuaremboensis". En:Tacuarembó, colección Los Departamentos, 15: 16-20. Montevideo, Banda Oriental.

INE (Instituto Nacional de Estadística). (2011). Resultados del Censo de Población 2011: población, crecimiento y estructura por sexo y edad. Recuperado de: http://www.ine.gub.uy/documents/10181/35289/analisispais.pdf\#targetText=La\%20poblaci\%C3\%B3n\%20total\%20contabilizada\%20a,es\%20de\%203.286.314\%20personas

Lasker, G. W. (1977). A coefficient of relationship by isonymy: a method for estimating the genetic relationship between populations. Human Biology, 49, 489-493.

Malécot, G. (1955). Decrease of relationship with distance, Cold Spring Harbour Symposyum, 20, 52-53.

Mantel, N. (1967). The detection of disease clustering and a generalized regression approach. Cancer Research, 27, 209-220.

Marenales, M y Luzuriaga, J.C. (1990). Los vascos en el Uruguay. Montevideo: Colección Nuestras Raíces, Banda Oriental.

Martínez Díaz, N. (1985). La emigración clandestina desde las Islas Canarias al Uruguay. Formas de incorporación social. Ensayo de estudio cuantitativo. En: V Coloquio de Historia Canario-Americana (1982), (pp.249-313). Las Palmas de Gran Canaria: Cabildo Insular de Gran Canaria.

Nei, M. (1973). The theory and estimation of genetic distance. En: Morton N.E. (Ed.), Genetic structure of populations (pp. 45-54), Honolulu: University Press of Hawaii.

OIM (Organización Internacional para las Migraciones). (2011). Perfil migratorio del Uruguay 2011. Recuperado de: http://cienciassociales.edu.uy/wp-content/uploads/sites/6/2014/11/Perfil-Migratorio-Uruguay-2011.pdf

Padrón Favre, O. (2011). El proceso de creación de los departamentos. En: Arocena F. (Ed.), Regionalización cultural del Uruguay (pp. 45-78), Montevideo: Universidad de la República.

Pellegrino A., Cabella W., Paredes M., Pollero R., Varela. C. (2008). De una transición a otra: la dinámica demográfica del Uruguay en el siglo XX. En: El Uruguay del siglo XX, tomo 3 (pp. 11-44). Montevideo, Banda Oriental.

Pi, R. y Vidart, D. (1969). El legado de los inmigrantes, I. Montevideo: Colección Nuestra Tierra, Banda Oriental.

Quagliotti, B. y Chamorro, V. (1977). Uruguay y su espacio. Antecedentes y una propuesta. Montevideo: Ed. Geosur.

Rodríguez-Larralde, A., Scapoli, C., Beretta, M., Nesti, C., Mamolini, E. y Barrai, I. (1998). Isonymy and the genetic structure of Switzerland. II Isolation by distance. Annals of Human Biology, 25, 533-540. doi:10.1080/03014469800006772

Rodríguez-Larralde, A., Morales, J. y Barrai, I. (2000). Surname frequency and the isonymy structure of Venezuela. American Journal of Human Biology, 12, 352-362. doi:10.1002/(SICI)15206300(200005/06)12:3<352::AID-AJHB5>3.0.CO;2-S

Rodríguez-Larralde, A., Scapoli, C.,Mamolini, E. y Barrai, I. (2007). Surnames in Texas: a population study through isonymy. Human Biology, 79, 215-239. doi:10.1353/hub.2007.0034

Rodríguez-Larralde, A. y Castro de Guerra, D. (2012). Inferencia de movimientos migratorios en los estados del oriente de Venezuela realizada a través de la distribución de apellidos. Interciencia, $37,349-354$.

Saitou, N. y Nei, M. (1987). The neighbor-joining method: a new method for reconstructing phylogenetic trees. Molecular Biology and Evolution, 4, 406-425. doi:10.1093/oxfordjournals.molbev.a040454 
Salzano, F.M. y Sans, M. (2014). Interethnic admixture and the evolution of Latin American populations. Genetics and Molecular Biology, 37(1 Suppl):151-170. doi:10.1590/s1415-47572014000200003

Sans, M. (2009). "Raza”, adscripción étnica y genética en Uruguay. RUNA Archivo para las Ciencias del Hombre, 30, 163-174. doi:https://doi.org/10.34096/runa.v30i2.771

Scapoli, C., Mamolini, E., Carrieri, A., Rodríguez-Larralde, A. y Barrai, I. (2007). Surnames in Western Europe: a comparison of the subcontinental populations through isonymy. Theoretical Population Biology, 71, 37-48. doi:10.1016/j.tpb.2006.06.010

Shi, Y., Li, L., Wang, Y., Chen, J., Yuan, Y. y Stanley, H. E. (2019a). Regional surname affinity: a spatial network approach. American Journal of Physical Anthropology, 168, 428-437. doi:10.1002/ajpa.23755

Shi, Y., Li, L., Wanga, Y., Chen, J., Stanley, H.E. (2019b). A study of Chinese regional hierarchical structure based on surnames. Physica A:Statistical Mechanics and its Applications, 518, 169-176. doi:10.1016/j. physa.2018.11.059

Smouse, P.E., Long, J.C. y Sokal, R.R. (1986). Multiple regression and correlation extensions of the Mantel test of matrix correspondence. Systematic Biology, 35, 627-632.

Sokal, R.R. y Michener, C.D. (1958). A statistical methods for evaluating relationships. University of Kansas Science Bulletin, 38, 1409-1448.

Tarskaia, L., Scapoli, C., Mamolini, E., Rodríguez-Larralde, A. y Barrai, I. (2009). Surnames in Siberia. A study of the population of Yakutia through isonymy. American Journal of Physical Anthropology, 138, 190-198. doi:10.1002/ajpa.20918

Tobler, W.R. (1970) A computer movie simulating urban growth in the Detroit region. Economic geography. Proceedings International Geographical Union. Commission on Quantitative Methods, 46, 234-240.

Veiga, D. (2015) Desigualdades sociales y territoriales en Uruguay. Montevideo: Departamento de Sociología, Universidad de la República.

Yagüe, J.L. y Díaz-Puente, J.M. (2008). Tres siglos de planificación regional en Uruguay: lecciones de experiencia para afrontar los retos de desarrollo en el siglo XXI. Estudios Geográficos (Montevideo), $264,247-280$.

Zei, G., Matessi, R.G., Siri, E., Moroni, A. y Cavalli-Sforza, L.L. (1983). Surnames in Sardinia: I. Fit of frequency distributions for neutral alleles and genetic population structure. Annals of Human Genetics, 47, 329-352. doi:10.1111/j.1469-1809.1983.tb01003.x 